\title{
Estudo comparativo das estruturas do segmento anterior de olhos de cães normais e com catarata, portadores ou não de Diabetes mellitus, avaliados por biomicroscopia ultrassônica ${ }^{1}$
}

\author{
Márcia P. Galego ${ }^{2 *}$, Angélica M.V. Safatle ${ }^{2}$, Denise Otsuki ${ }^{3}$, Ana P. Hvenegaard ${ }^{2}$, \\ Vera R. Castanheira ${ }^{4}$ e Paulo S.M. Barros ${ }^{2}$
}

\begin{abstract}
Galego M.P., Safatle A.M.V., Otsuki D., Hvenegaard A.P., Castanheira V.R. \& Barros P.S.M. 2012. [Comparison of anterior ocular segment structures in healthy dogs, with diabetic or no diabetic cataract, by ultrasound biomicroscopy.] Estudo comparativo das estruturas do segmento anterior de olhos de cães normais e com catarata, portadores ou não de Diabetes mellitus, avaliados por biomicroscopia ultrassônica. Pesquisa Veterinária Brasileira 32(1):66-71. Departamento de Cirurgia, Faculdade de Zootecnia e Medicina Veterinária, Universidade de São Paulo, Av. Prof. Dr. Orlando de Marques de Paiva 87, Bloco 8 superior, Cidade Universitária, São Paulo, SP 05508-270, Brazil. E-mail: mpgalego@uol.com.br

Cataracts represent the leading cause of blindness in dogs. The second most common cause of cataract in dogs is a result of metabolic alterations caused by Diabetes mellitus (DM). Ultrasound biomicroscopy (UBM) is a high-frequency (50 MHz) ultrasonographic method that produces B mode images of microscopic quality. The objective of this study was, by means of UBM use, to compare the anterior segment structures of the canine eyes, both with diabetic and non-diabetic cataract, in order to detect changes caused by DM. The parameters evaluated were: cornea thickness, anterior chamber's depth, increased cellularity inside the anterior chamber, and iridocorneal angle measurement. Eighty-seven eyes of 47 dogs were examined, divided into three groups: control (GCO), non-diabetic cataract (GCAT) and diabetic cataract (GDM). The results showed that the diabetic group presented a higher cornea thickness than the other groups. The control group showed deeper anterior chambers without increased cellularity. When the iridocorneal angle measurements were analyzed, it was found that there were no statistically significant differences between the three groups. Based on these results, we can conclude that: the eyes of diabetic dogs with cataract showed a central cornea higher thickness compared to the eyes of dogs with cataract of different etiologies, and to dogs with normal eyes; there is a decrease of the anterior chamber depth and a increase of cellularity in the eyes of dogs with cataract compared to normal eyes, there is no significant difference between the iridocorneal angle measurement in the eyes of dogs with cataract, diabetic or not, and normal dogs.
\end{abstract}

INDEX TERMS: Ultrasound biomicroscopy, dogs, cataract, Diabetes mellitus.

RESUMO.- A catarata representa uma das principais causas de cegueira em cães, sendo que as alterações metabólicas provocadas pelo Diabetes mellitus (DM), constituem a segun-

\footnotetext{
${ }^{1}$ Recebido em 24 de janeiro, de 2011.

Aceito para publicação em 28 de junho de 2011.

${ }^{2}$ Serviço de Oftalmologia, Departamento de Cirurgia, Faculdade de Medicina Veterinária e Zootecnia, Universidade de São Paulo (USP), Av. Prof. Dr. Orlando Marques de Paiva, 87, Cidade Universitária, São Paulo, SP 05508- 270, Brasil. * Autor para correspondência: mpgalego@uol.com.br

${ }^{3}$ Laboratório de Investigação Médica (LIM08), Faculdade de Medicina (FM), Universidade de São Paulo (USP), Av. Dr. Arnaldo 455, Cerqueira César, São Paulo, SP 01246-903, Brasil.

${ }^{4}$ Departamento Oftalmologia, Hospital das Clínicas, FM-USP, São Paulo, SP.
}

da causa mais comum de catarata nesta espécie. A biomicroscopia ultrassônica (UBM) é um método ultrassonográfico de alta frequência $(50 \mathrm{MHz})$ que permite a obtenção de imagens do modo B de qualidade microscópica. Este estudo objetivou, por meio do uso da UBM, comparar as estruturas do segmento anterior de olhos de cães com catarata, diabética e não-diabética, às de cães normais, para verificar possíveis alterações decorrentes do DM. Os parâmetros avaliados foram espessura da córnea, profundidade da câmara anterior, aumento de celularidade no interior da câmara anterior e medida do ângulo iridocorneal. Foram realizados exames de 87 olhos de 47 animais da espécie canina, divididos em 3 grupos: grupo controle (GCO), grupo de portadores de catarata 
não-diabéticos (GCAT) e o grupo dos diabéticos (GDM). Os resultados revelam que o grupo dos diabéticos apresentou maiores espessuras de córnea que os demais grupos enquanto o grupo controle apresentou maiores câmaras anteriores. Encontrou-se aumento de celularidade em câmara anterior apenas nos grupos com catarata. Quando analisadas as medidas do ângulo iridocorneal, não houve diferença entre os 3 grupos. Com base no que foi aferido, permite-se concluir que olhos de cães diabéticos com catarata apresentam maior espessura de córnea central que olhos de cães com catarata de outras etiologias e de cães normais, que há diminuição da câmara anterior, com aumento de celularidade, em olhos de cães com catarata, quando comparados a cães normais e que não há diferença na medida do ângulo iridocorneal em olhos de cães com catarata, diabética ou não, e de cães normais.

TERMOS DE INDEXAÇÃO: Biomicroscopia ultrassônica, cães, catarata, Diabetes mellitus.

\section{INTRODUÇ̃̃O}

A catarata é a opacidade do cristalino ou das suas cápsulas e representa uma das principais causas de cegueira em cães. A causa mais frequente de desenvolvimento de catarata em cães é alteração hereditária no metabolismo proteico da lente, seguida pelas alterações metabólicas provocadas pelo Diabetes mellitus (Glover \& Constantinescu 1997).0 Diabetes mellitus (DM) é uma das endocrinopatias mais prevalentes em cães, caracterizada pela absoluta ou relativa deficiência de insulina e, ao alterar o equilíbrio osmótico no interior da lente, leva à catarata bilateral, simétrica e aguda, sendo a espécie canina particularmente suscetível ao seu desenvolvimento (Basher \& Roberts 1995). Aos 16 meses de diagnóstico, 80\% dos cães diabéticos apresentam catarata (Beam et al. 1999), essa progride de forma rápida e osmoticamente ativa resultando em intumescência da lente e processo inflamatório, uveíte facolítica.

A biomicroscopia ultrassônica (UBM) é um método ultrassonográfico de alta frequência $(50 \mathrm{MHz})$, que permite obter de imagens do modo $B$ de qualidade microscópica. A resolução axial de $37 \mathrm{~mm}$, dez vezes maior que o ultrassom convencional, e a penetração limitada de cerca de 5 $\mathrm{mm}$ tornam o método adequado para exame do segmento anterior do bulbo ocular mesmo na presença de opacidade dos meios. A córnea, câmara anterior, ângulo iridocorneal, íris, câmara posterior, zônula, corpo ciliar, esclera anterior e, eventualmente, coróide, assim como a cápsula anterior e parte do equador do cristalino, podem ser avaliadas em tempo real (Pavlin et al. 1991, Liebmann \& Ritch 1996, Castanheira 2003).

Este estudo buscou, por meio do uso da UBM, avaliar e comparar as estruturas do segmento anterior de olhos de cães normais e com catarata, diabética e não-diabética, verificando possíveis alterações decorrentes do DM.

\section{MATERIAL E MÉTODOS}

Foram realizados exames de 87 olhos de 47 animais da espécie canina, 19 machos e 28 fêmeas de raças variadas e idade entre 02 e 14 anos, divididos em 3 grupos:

- 0 grupo controle (GCO), composto 10 cães hígidos, sem catarata e sem Diabetes mellitus, sendo 4 machos e 6 fêmeas, com idades de 2-14 anos. Deste grupo foram utilizados no estudo os exames de 18 olhos, 8 direitos e 10 esquerdos.

- 0 grupo dos portadores de catarata não-diabéticos (GCAT), com 20 animais, composto por 9 machos e 11 fêmeas, com idades de 2-12 anos. Foram incluídos no estudo exames de 38 olhos, 19 esquerdos e 19 direitos.

- 0 terceiro grupo, grupo dos diabéticos (GDM), composto por 17 animais com catarata, portadores de Diabetes mellitus, sendo 06 machos e 11 fêmeas, com idades de 7-14 anos. Utilizaram-se os exames de 15 olhos direitos e 16 olhos esquerdos, totalizando 31 olhos.

Todos os animais foram submetidos a exames físico, laboratoriais e oftalmológico completo antes de serem incluídos no estudo, incluindo tonometria ${ }^{5}$ e oftalmoscopia indireta ${ }^{6}$, quando possível. Aqueles que apresentaram outras oftalmopatias foram excluídos do estudo.

Para a contenção foi administrada atropina ${ }^{7}(0,044 \mathrm{mg} / \mathrm{kg})$ por via subcutânea e, após 15 minutos, xilazina ${ }^{8}(0,5 \mathrm{mg} / \mathrm{kg})$ e butorfanol $^{9}(0,2 \mathrm{mg} / \mathrm{kg})$ por via intramuscular.

Instilou-se colírio de tropicamida $1 \%{ }^{10}$, seguido de colírio de fenilefrina $10 \%{ }^{11}$ com intervalo de 5 minutos entre eles em todos os olhos, portanto, todos os animais se encontravam em midríase medicamentosa durante a realização do exame.

A UBM foi realizada em aparelho "Paradigm Ultrasound Biomicroscope"12 modelo P40, equipado com transdutor de $50 \mathrm{MHz}$, com velocidade de onda de $1540 \mathrm{~m} / \mathrm{s}$, penetração efetiva de aproximadamente $5 \mathrm{~mm}$, o campo de $5,5 \times 5,5 \mathrm{~mm}$ e resolução de $50 \mathrm{~mm}$. Os animais foram posicionados em decúbito lateral (Fig.1) e o olho a ser examinado recebeu colírio anestésico ${ }^{13}$ e um cone acrílico transparente (eye cup ${ }^{14}$ ) que, uma vez adaptado à fenda palpebral (Fig.2), foi preenchido com cerca de $3 \mathrm{ml}$ de solução viscoelástica ${ }^{15}$ e solução fisiológica a $0,9 \%{ }^{16}$, evitando-se bolhas de ar na mistura.

0 transdutor da sonda do aparelho, que é exposto, foi parcialmente imerso na solução contida pelo eye-cup e mantido de forma perpendicular (Fig.3.) a cerca de $1 \mathrm{~mm}$ da superfície do olho a ser examinado produzindo imagens da área de interesse. Na obtenção das imagens, manteve-se o ganho de $80 \mathrm{~dB}$, exceto na córnea, para qual se utilizou $60 \mathrm{~dB}$.

Captou-se em todos os grupos, imagens da córnea na sua porção central e da câmara anterior, na sua porção central e periférica, mantendo o ângulo iridocorneal no centro da imagem, sendo essas

\footnotetext{
${ }^{5}$ Tono-pen.XL, BIO-RAD, 2000 Alfred Nobel Drive, Hercules, CA 94547, EUA.

${ }^{6}$ Oftalmoscópio binocular indireto, IO-H, Neitz Instruments Corporation Ltd, 36-13 Wakamatsu-Cho, Shinjuku-Ku, Tokyo 162-0056, Japão.

${ }^{7}$ Atropion $0.50 \mathrm{mg}{ }^{\circledR}$, Ariston Indústrias Químicas e Farmacêuticas Ltda., Rua Adherbal Stresser 84, Jd. Arpoador, São Paulo, SP 05566-000.

${ }^{8}$ Xilazin $2 \% ®$, Syntec do Brasil Ltda, Rua Soluções do lar 105, Cotia, SP 06716-020.

${ }^{9}$ Torbugesic 10 mg®, Fort Dodge Saúde Animal Ltda., Rua Luiz Fernando Rodriguez 1701, Vl. Boa Vista, Campinas, SP 13065-858.

${ }^{10}$ Mydriacyl 1\%®, Alcon Laboratórios do Brasil Ltda, Av. Nossa Senhora Assunção 736, Vl. Butantã, São Paulo, SP 05359-001.

${ }^{11}$ Fenilefrina $10 \% ®$, Allergan Produtos farmacêuticos Ltda, Av. Guarulhos, 3180, Guarulhos, SP 07030-000.

12 Paradigm Ultrasound Biomicroscope, Paradigm Medical Industries Inc., 4273 South 590 West Salt Lake City,UT 84123, EUA.

${ }^{13}$ Anestalcon $0.5 \% ®$, Alcon Laboratórios do Brasil Ltda, Av. Nossa Senhora Assunção 736, Vl. Butantã, São Paulo, SP 05359-001.

${ }^{14}$ Eye cup, Paradigm Medical Industries Inc., 4273 South 590 West, Salt Lake City,UT 84123, EUA.

${ }^{15}$ Metilcelulose $2 \% ®$, Ophthalmos Indústria Farmacêutica, Rua Nhandirobas 471, São Paulo, SP 04349-030.

${ }^{16}$ Cloreto de Sódio 0,9\%®, Samtec Biotecnologia, Rua General Augusto Soares dos Santos, 465, Lagoinha, Ribeirão Preto, SP 14095-240.
} 


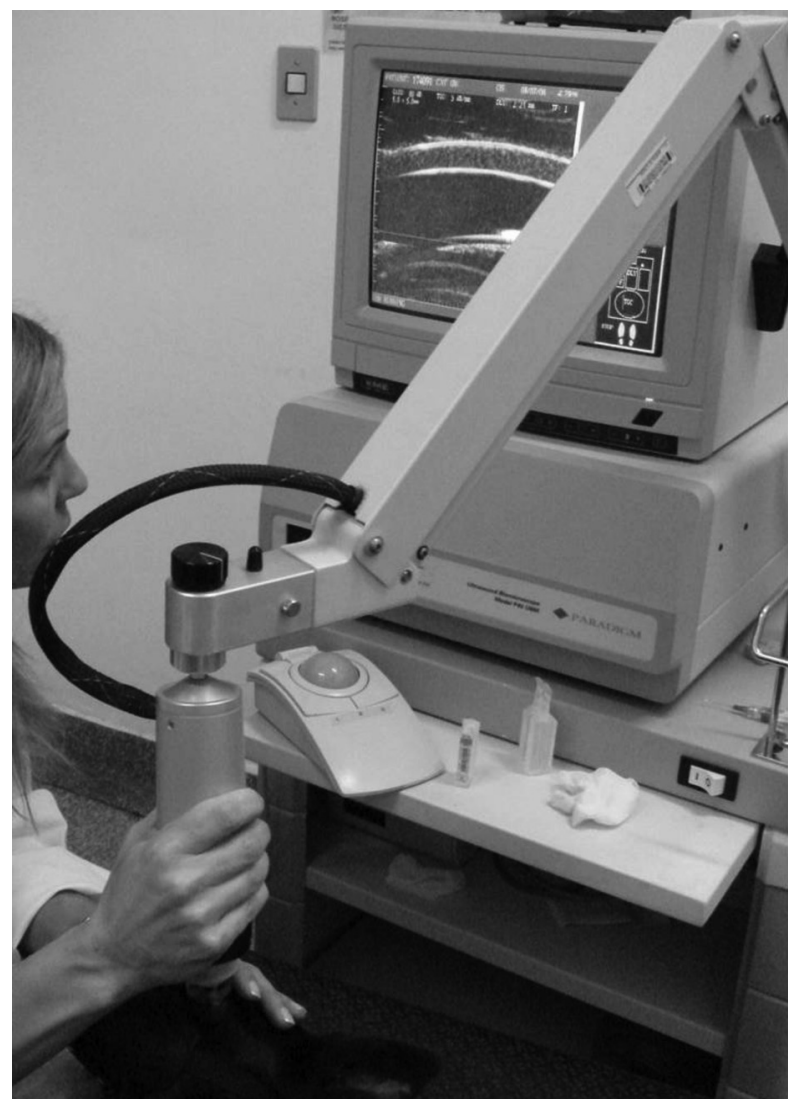

Fig.1. Aparelho Paradigm Ultrasound Biomicroscope modelo P40 durante a realização do exame em um cão SRD, fêmea, 11 anos com catarata diabética. Observar o posicionamento do animal em decúbito lateral direito com a cabeça levemente inclinada para cima para a correta realização do exame nos quadrantes analisados.

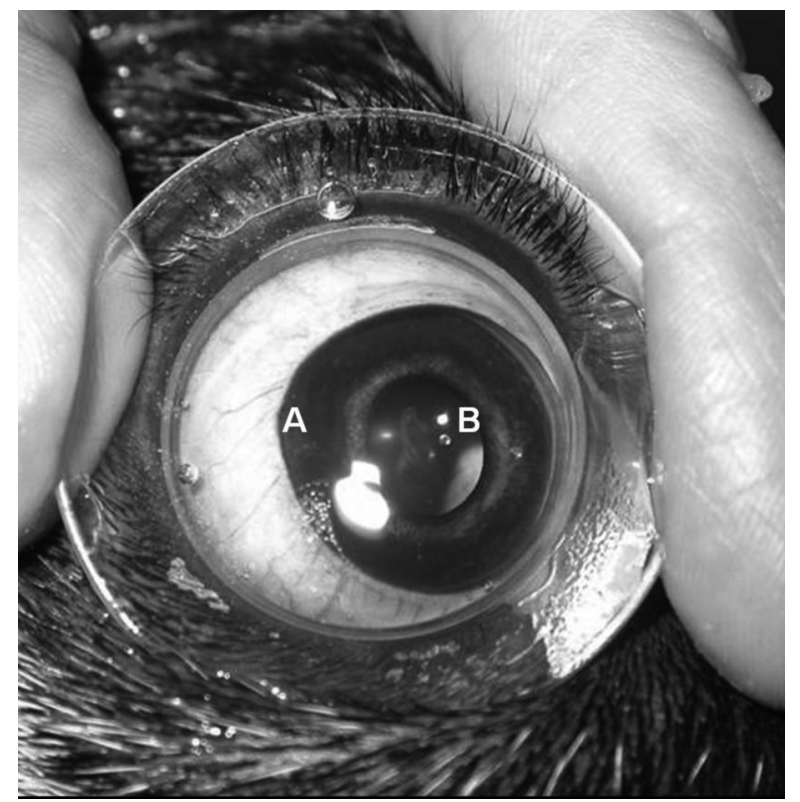

Fig.2. Adaptação do eye-cup à fenda palpebral do olho direito de cão SRD, 7 anos, sem alteração ocular. Observar a completa exposição da área que será avaliada pela UBM: (A) ângulo irido-corneal e (B) espessura central da córnea; profundidade e presença de células inflamatórias na câmara anterior.

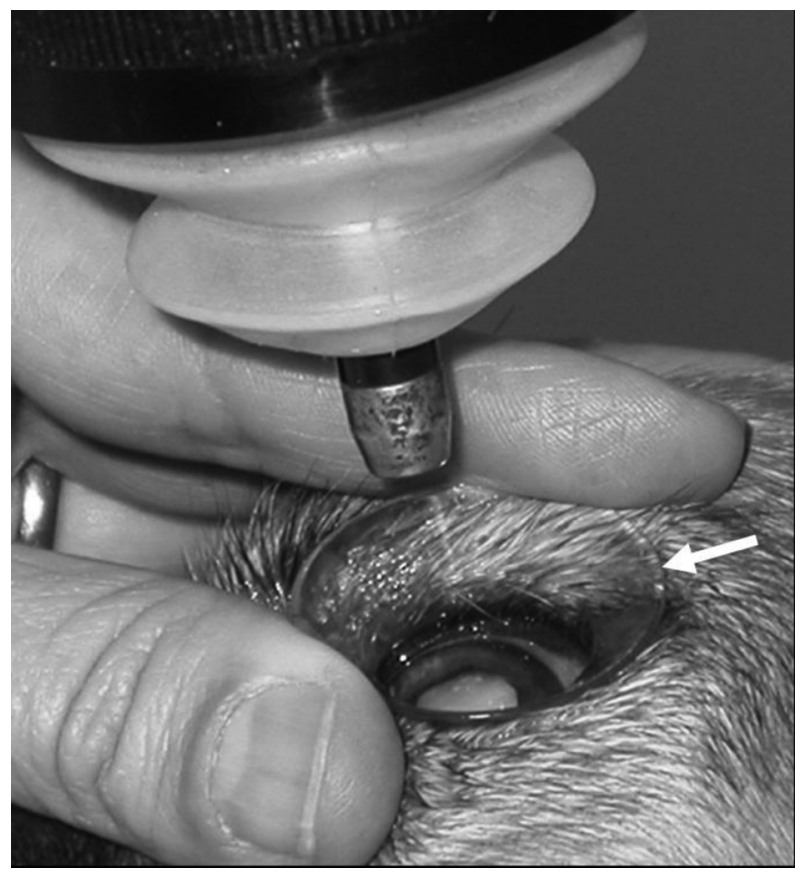

Fig.3. Transdutor do UBM posicionado sobre o eye cup, indicado pela seta branca, em olho esquerdo de cão SRD, 9 anos, com catarata hipermadura.

obtidas no quadrante temporal-superior, correspondente das 9 às 12 horas, no olho direito e, das 12 às 3 horas, no olho esquerdo. As imagens foram armazenadas para posterior análise e impressão.

Após a captação das imagens, avaliou-se a espessura da córnea e a profundidade da câmara anterior, mensurando-se a distância em milímetros entre os picos, obtidos pelo modo A, projetados nas imagens modo B. Na abertura do ângulo iridocorneal considerou-se a medida em graus, disponível pela função caliper do aparelho. A presença de ecos puntiformes na câmara anterior, indicativa do aumento de celularidade no interior da mesma, foi avaliada qualitativamente como presente ou ausente.

\section{Parâmetros analisados}

Foram estudadas, nos olhos dos cães da amostra, as seguintes variáveis (Fig.4.):

- Espessura central da córnea: determinada pela superfície corneana anterior e posterior, na região central.

- Profundidade da câmara anterior dos olhos: distância entre a superfície posterior da córnea e a cápsula anterior do cristalino, na região central pupilar.

- Ângulo iridocorneal: formado pela medida em graus do cruzamento de segmentos de retas passando entre a porção interna da córnea e esclera e a porção anterior da superfície da íris.

- Presença de células da câmara anterior dos olhos: pesquisa de pontos hiperecóicos na câmara anterior, indicativos de celularidade em seu interior, de forma qualitativa, como presentes ou ausentes.

\section{RESULTADOS}

Os valores encontrados para a espessura da córnea variaram de $0,405 \mathrm{~mm}$ a $0,764 \mathrm{~mm}$. No grupo GCO a média dos valores obtidos para este parâmetro foi $0,565 \mathrm{~mm}$, com DP de $0,045 \mathrm{~mm}$ e mediana de $0,573 \mathrm{~mm}$; no GDM, média de $0,615 \mathrm{~mm}$, com DP de $0,067 \mathrm{~mm}$ e mediana de $0,613 \mathrm{~mm}$ e no GCAT, média de $0,570 \mathrm{~mm}$, com DP de $0,08 \mathrm{~mm}$ e mediana de $0,564 \mathrm{~mm}$. Desta forma, o grupo dos diabéticos com catarata 

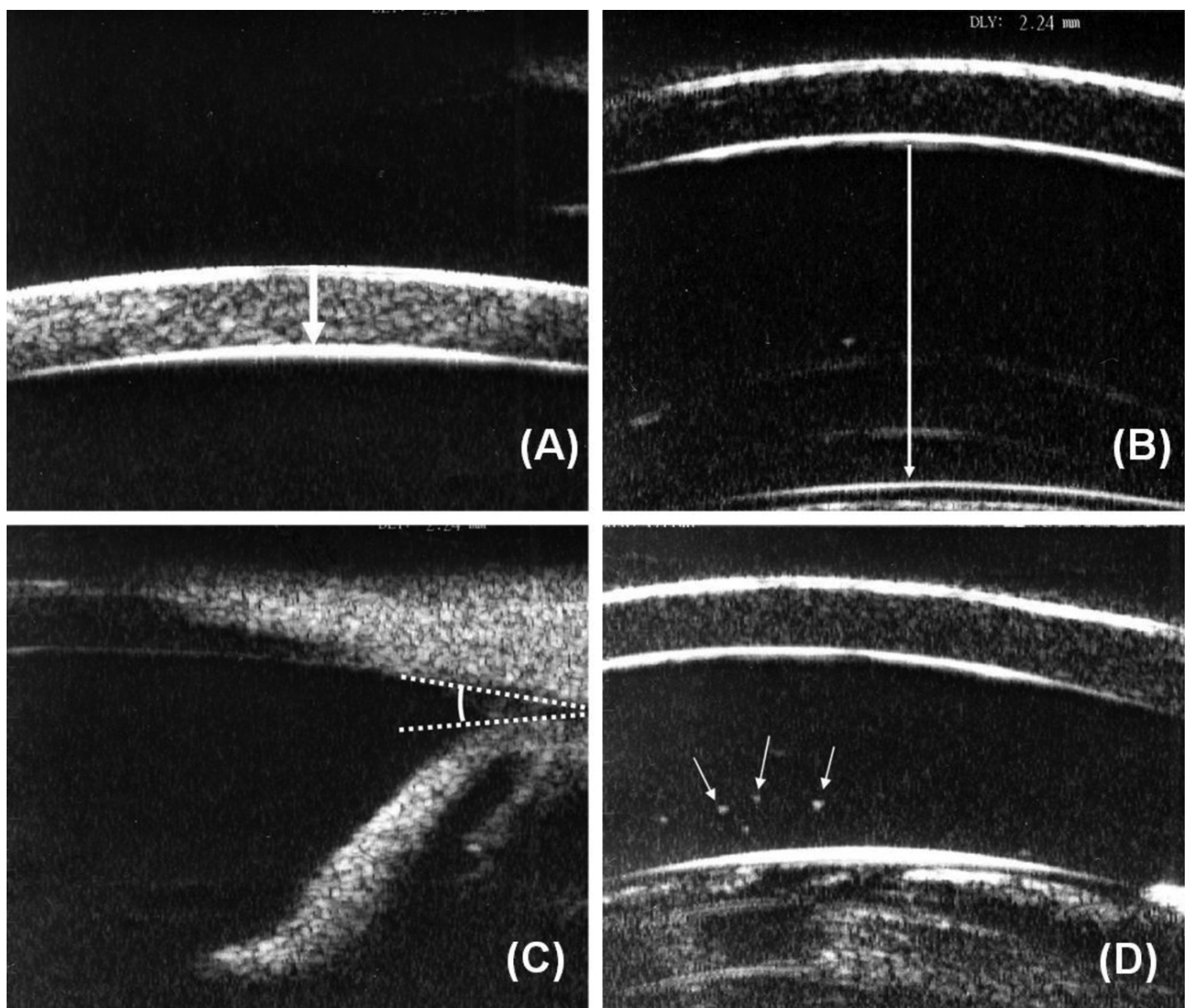

Fig.4. Imagens das diferentes variáveis avaliadas durante o estudo: (A) Aspecto da córnea central na UBM, indicada pela seta branca, ilustrando a distância da superfície anterior à superfície posterior da córnea. (B) Aspecto da câmara anterior na UBM. A seta branca ilustra a distância da superfície posterior da córnea à cápsula anterior do cristalino. (C) Esquema de medida do ângulo iridocorneal, indicada pelas linhas tracejadas. (D) Imagem da câmara anterior com presença de pontos hiperecóicos (células inflamatórias) em seu interior (setas).

(GDM) apresentou maiores espessuras de córnea que os demais grupos, com diferença estatisticamente significante com os demais grupos (GCO e GCAT).

Quanto à profundidade central de câmara anterior, os valores variaram entre $1,887 \mathrm{~mm}$ e $4,716 \mathrm{~mm}$. 0 grupo controle (GCO) apresentou média de 3,863mm com DP de 0,188mm e mediana de 3,866mm; no GDM média foi de 3,456mm, DP de

Quadro 1. Valores obtidos segundo os parâmetros analisados para os diferentes grupos

\begin{tabular}{lccc}
\hline & \multicolumn{3}{c}{ Média (desvio padrão) } \\
\cline { 2 - 4 } & GCO & GCAT & GDM \\
\hline \multirow{2}{*}{ Espessura corneal } & $0,565 \mathrm{~mm}$ & $0,570 \mathrm{~mm}$ & $0,615 \mathrm{~mm}$ \\
& $(0,045)$ & $(0,08)$ & $(0,067)^{\mathrm{a}}$ \\
Profundidade da câmara & $3,863 \mathrm{~mm}$ & $3,467 \mathrm{~mm}$ & $3,456 \mathrm{~mm}$ \\
anterior & $(0,188)^{\mathrm{b}}$ & $(0,475)$ & $(0,810)$ \\
Ângulo irido corneal & $28,87^{\circ}$ & $27,24^{\circ}$ & $26,08^{\circ}$ \\
& $(6,55)$ & $(5,64)$ & $(6,28)$ \\
\% de cães que apresentaram & $0 \%^{\mathrm{c}}$ & $34,20 \%$ & $29 \%$ \\
celularidade em câmara anterior & & &
\end{tabular}

0,810 mm e mediana de 3,49mm e no GCAT possui média de $3,467 \mathrm{~mm}$, DP de $0,475 \mathrm{~mm}$ e mediana de $3,377 \mathrm{~mm}$. 0 grupo controle apresentou maiores valores de profundidade de câmara anterior e há diferença estatisticamente significante entre GCO e GCAT.

Os valores obtidos na mensuração do ângulo iridocorneal se situam entre $14,25^{\circ}$ e $45,48^{\circ}$. Não houve diferença estatisticamente significante entre os 3 grupos. A média dos valores obtidos para GCO foi $28,87^{\circ}$ com DP de $6,55^{\circ}$ e mediana de $27,44^{\circ}$; para GDM, média $26,08^{\circ}$ e DP de $6,28^{\circ}$ e mediana de $25,78^{\circ}$ e para GCAT, média $27,24^{\circ}$, DP de $5,64^{\circ}$ e mediana de $28,1^{\circ}$.

Não houve registro da presença de células no grupo controle, porém, estas foram encontradas em 34,2\% dos cães com catarata não diabética e em $29 \%$ dos cães diabéticos. Há portanto, diferença estatisticamente significante entre o GCO e os demais (Quadro 1).

\section{DISCUSSÃO}

A UBM não é um procedimento doloroso e é realizado, rotineiramente, em pacientes humanos apenas com a instilação de colírio anestésico. Em animais é necessária a

${ }^{b}$ Diferente de GCAT: $\mathrm{p}<0,05$.

${ }^{c}$ Diferente dos grupos GCAT e GDM: $\mathrm{p}<0,05$. 
contenção para permitir o posicionamento do paciente, sua imobilização e diminuir seu estresse. Uma vez que não há estímulo doloroso durante a execução do exame, optou-se pela sedação, que promove grau moderado de depressão do sistema nervoso central mantendo o animal acordado, porém calmo, de tal forma que, mediante a estímulo, este pudesse ser despertado desse estado (Fantoni \& Cortopassi 2002).

A adaptação do cone acrílico (eye cup) à fenda palpebral é fator limitante nas diferentes espécies. Nos humanos, afecções que prejudiquem a abertura palpebral, como simbléfaro, tumores palpebrais e blefarospasmo podem impedir a realização do exame de UBM (Allemann 2000). Nos cães, especialmente os dolicocefáficos de pequeno porte, a abertura da fenda palpebral por vezes é insuficiente, aumentando a dificuldade ou mesmo impossibilitando a realização do exame (Gibson et al. 1998). Sugere-se o desenvolvimento de cones menores ou o uso de um meio viscoelástico sem o cone para o exame em felinos (Aubin et al. 2003). Apesar disso, estudo recente descreveu que o transdutor também pode ser colocado diretamente sobre o bulbo ocular, sem alusão à cuba de imersão (Rose et al. 2008). Na execução dos exames deste estudo, a adaptação do eye cup em alguns cães foi bastante difícil, especialmente naqueles com menores fendas palpebrais, já que estes foram desenvolvidos para uso humano. 0 desenvolvimento de diferentes cubas de imersão ou de técnicas que as dispensse, como os novos aparelhos de alta frequência que utilizam sondas seladas que permitem a avaliação com amplo campo e transpalpebral, aumenta a aplicabilidade da UBM na medicina veterinária.

Nos cães, a proximidade da rima palpebral e da terceira pálpebra dificultam a obtenção de imagens nos quadrantes ventrais e nasais (Gibson et al. 1998), o que já foi observado por autores que realizaram um estudo com corte ultrassonográfico apenas na altura do limbo dorsal (Rose et al. 2008), e outros que relataram a dificuldade na obtenção de imagens muito periféricas nos aspectos ventral, nasal e temporal dos olhos examinados (Maia 2003). Encontrou-se limitação no exame das porções nasal e ventral, tanto pela conformação anatômica das pálpebras quanto pela impossibilidade de movimentação do olho. Assim, padronizou-se neste estudo realizar as medidas no quadrante temporal-dorsal.

Os achados do exame ocular pela UBM podem ser alterados pelo uso de midriáticos, inclusive quando associados a alguns anestésicos tópicos, por modificar a anatomia das estruturas anteriores e sua resposta aos estímulos (Castanheira 2003). Medidas do ângulo iridocorneal em olhos de cães, portadores e não portadores de catarata, foram comparadas com auxílio do UBM antes e após a dilatação pupilar. Na comparação entre os grupos, não foram encontradas diferenças estatisticamente significantes, antes e após a midríase. Quando os resultados do grupo com catarata foram comparados antes e após a midríase não houve diferença estatísticamente significante, entretanto, no grupo sem catarata, as medidas do ângulo foram significativamente menores após a dilatação. (Rose et al. 2008).

No presente estudo, considerando-se que todos os exames foram realizados rigorosamente sob as mesmas condições, acredita-se que a midríase medicamentosa não interferiu na comparação entre os grupos, objetivo deste trabalho.
0 grupo de catarata diabética (GDM) apresentou maiores espessuras de córnea central, com diferença estatisticamente significante quando comparado aos demais grupos. Os valores encontrados para espessura da córnea central no GCO (média de $0,565 \mathrm{~mm}$ com DP de $0,045 \mathrm{~mm}$ ) são próximos aos obtidos em estudo semelhante $0,550 \pm 0,33 \mathrm{~mm}$ (Maia 2003).0 resultado é consistente com a constatação de que o endotélio do cão diabético apresenta anormalidades como polimegatismo e pleomorfismo, que leva ao edema estromal e consequente espessamento da córnea, diretamente correspondente ao nível de controle glicêmico (Yee et al. 1985). Embora o mecanismo de lesão endotelial não esteja totalmente elucidado, é possível que a aldose redutase interfira no metabolismo das células endoteliais causando a lesão (Datiles et al. 1990).

Observou-se com o auxílio da paquimetria ultrassônica que cães diabéticos apresentam maior espessura central da córnea quando comparado aos não-diabéticos (Lynch \& Brinkis 2006). Acredita-se que a uveíte, que frequentemente acompanha o rápido aparecimento da catarata diabética no cão, também desempenhe papel importante na patofisiologia desta alteração, provocando edema, ainda que subclínico.

Um estudo, que mediu a profundidade da câmara anterior em olhos de cães sem catarata antes e após a facoemulsificação com implante de LIO, encontrou o valor médio de 3,83mm no pré-operatório (Maia 2003). Estes valores são próximos aos obtidos neste estudo nos olhos do grupo controle (GCO), em que a média foi de $3,86 \mathrm{~mm}$ com DP $0,188 \mathrm{~mm}$.

A mensuração do ângulo da câmara anterior em humanos por UBM, é feita por um segmento de reta, a partir esporão escleral, perpendicular à face interna da esclera e da córnea e outro passando na superfície da raiz íris. Para olhos normais, encontram-se valores de $30^{\circ}\left(\mathrm{DP} 11^{\circ}\right)$. Como a abertura do ângulo pode variar de acordo como a abertura pupilar, a reprodutibilidade depende da documentação precisa da posição radial (usualmente em horas) em que foi realizada a medida, da localização adequada do esporão escleral, da manutenção das condições de iluminação da sala e da midríase pupilar (Pavlin \& Foster 1995).

0 cão não possui esporão escleral e ainda não se padronizou a forma de medir o ângulo por UBM nesta espécie, o que dificulta, ou até mesmo impede, a comparação dos resultados entre os diferentes trabalhos. No presente trabalho determinamos que a medida do ângulo em graus seria obtida de maneira análoga a dos humanos, de tal forma que o ápice foi colocado no sulco iriano com um segmento de reta passando pela face interna da esclera e outro pela face anterior da íris.

No grupo controle (GCO), o valor médio obtido foi $28,87^{\circ} \pm 6,5^{\circ}$, próximo ao encontrado em estudo realizado em cães da raça Cocker Spaniel sem catarata, com média de $26,2^{\circ} \pm 4,5^{\circ}$ (Gibson et al. 1998). Porém, outro estudo em cães sem catarata, refere o valor médio do ângulo antes da dilatação pupilar de $10,4^{\circ} \pm 5,0^{\circ}$ e, após a dilatação, $12,6^{\circ} \pm 5,3^{\circ}$ (Rose et al. 2008). Num relato de caso, de implante de válvula de Ahmed por elevação de pressão intra-ocular (PIO) pós facoemulsificação e implante de lente intra-ocular (LIO) (Garciá-Sanches et al. 2005), o ângulo é mensurado em $30^{\circ}$ após a facoemulsificação e classificado como estreito. 
Em olhos com catarata, um estudo refere o valor médio do ângulo de $13,6^{\circ} \pm 6,4^{\circ}$ antes e $12,8^{\circ} \pm 5,8^{\circ}$ após a midríase (Rose et al. 2008). Esses valores diferem bastante dos obtidos no presente estudo, tanto no GCAT, cuja média foi de $27,24^{\circ} \pm 5,64^{\circ}$, quanto no GDM, com média de $26,08^{\circ} \pm 6,28^{\circ}$, ambos obtidos após dilatação pupilar.

A presença de células em câmara anterior nos grupos com catarata, diabéticos ou não, e sua ausência no grupo controle se explica pela frequente ocorrência de uveíte induzida pela lente no cão, que pode ser encontrada em todos os estágios de maturação da catarata nesta espécie, ainda que de forma subclínica (Hendrix 2007).

\section{CONCLUSÕES}

Com base no que foi exposto, podemos concluir que:

- Olhos de cães diabéticos com catarata apresentam maior espessura de córnea central que olhos de cães com catarata de outras etiologias e de cães normais.

- Há diminuição da câmara anterior em olhos de cães com catarata não diabética, quando comparados a cães normais.

- Há aumento de celularidade em câmara anterior em olhos de cães com catarata, quando comparados a olhos de cães sem catarata.

- Não há diferença na medida do ângulo iridocorneal em olhos de cães com catarata, diabética ou não, e de cães normais.

Agradecimentos.- À FAPESP pelo apoio financeiro (Proc. 00/12137-0).

\section{REFERÊNCIAS}

Allemann N. 2000. Biomicroscopia ultra-sônica (UBM) ou ultra-sonografia de alta freqüência. Páginas de Actualización en Oftalmologia, n.2. Disponível em <http://www.oftalmologos.org.ar/publicaciones/alleman.html> Acesso em 25 set. 2008.

Aubin M.L., Powell C.C., Gionfriddo J.R. \& Fails A.D. 2003. Ultrasound biomicroscopy of the feline anterior segment. Vet. Ophthalmol. 6(1):15-17.

Basher A.W. \& Roberts S.M. 1995. Ocular manifestation of Diabetes mellitus: diabetic cataract in dogs. Vet. Clin. North Am., Small Anim, Pract, 25(3):661-676.

Beam S., Correa M.T. \& Davidson M.G. 1999. A retrospective-cohort study on the development of cataracts in dogs with Diabetes mellitus: 200 cases. Am. Coll. Vet. Ophthalmol. 2:169-172.
Castanheira V.R.C. 2003. Biomicroscopia ultra-sônica, p.183-208. In: Betinjane A.J \& Carani J.C.E. (Eds), Ecografia Ocular e Orbitária. Roca, São Paulo.

Datiles M.B., Kador P.F., Kashima K., Kinoshita J.H. \& Sinha A.1990. The effects of sorbinil, an aldose reductase inhibitor, on the corneal endothelium in galactosemic dogs. Invest. Ophthalmol. Vis. Sci. 31(11):2201-2204.

Fantoni D.T. \& Cortopassi S.G. 2002. Medicação Pré-Anestésica, p.151-158. In: Ibid. (Eds), Anestesia em Cães e Gatos. Roca, São Paulo.

Garciá-Sanchez G.A., Whitley R.D., Brooks D.E., Trigo F. \& Piñón A. 2005. Ahmed valve implantation to control intractable glaucoma after phacoemulsification and intraocular lens implantation in a dog. Vet. Ophthalmol. 8(2):139-144.

Gibson T.E., Roberts S.M., Severin G.A., Steyn P.F. \& Wrigley R.H. 1998. Comparison of gonioscopy and ultrasound biomicroscopy for evaluating the iridocorneal angle in dogs. J. Am. Vet. Med. Assoc. 213(5):635-638.

Glover T.D. \& Constantinescu G.M. 1997. Surgery for cataracts. Vet. Clin. North Am., Small Anim. Pract. 27(5):1143-1173.

Hendrix D.V.H. 2007. Diseases and surgery of the canine anterior uvea, p.355-437. In: Gelatt K.N. (Ed.), Veterinary Ophthalmology. $4^{\text {th }}$ ed. Blackwell Publishing, Iowa.

Liebmann, J.M. \& Ritch R. 1996. Ultrasound biomicroscopy of the anterior segment. J. Am. Optom. Assoc. 67(8):469-479.

Lynch G.L. \& Brinkis J.L. 2006. The effect of elective phacofragmentation on central corneal thickness in dog. Vet. Ophthalmol. 9(5):303-310.

Maia F.B.N. 2003. Biomicroscopia ultra-sônica (UBM) do segmento anterior ocular em cães submetidos a facoemulsificação experimental com e sem implante de lente intra-ocular (LIO). Dissertação de Mestrado em Medicina Veterinária, Faculdade de Medicina Veterinária e Zootecnia, USP, São Paulo, SP. 86p.

Pavlin C.J. \& Foster F.S. 1995. Ultrasound Biomicroscopy of the Eye. Springer-Verlag, New York. 208p.

Pavlin C.J., Harasiewicz K., Sherar M.D. \& Foster F.S. 1991. Clinical use of ultrasound microscopic. Ophthalmology 989(3):287-295.

Rose M.D., Mattoon J.S., Gemensky-Metzler A.J., Wilkie D.A. \& Rajala-Schultz P.J. 2008. Ultrasound biomicroscopy of the iridocorneal angle of the eye before and after phacoemulsification and intraocular lens implantation in dogs. Am. J. Vet. Res. 69(2):279-288.

Schwartz D.E. 1974. Corneal sensitivity in diabetics. Arch. Ophthal. 91:174178.

Williams D.L. 2004. Lens morphometry determined by B-mode ultrasonografy of the normal and cataractous canine lens. Vet. Ophthalmol. 7(2):91-95.

Yee R.W., Matsuda M., Kern T.M., Engerman R.L. \& Edelhauser H.F. 1985. Corneal endothelial changes in diabetics dogs. Curr. Eye Res. 4(7):759766. 